\title{
Aspectos éticos en la investigación cualitativa con niños
}

\author{
Ethical aspects in qualitative research with \\ children
}

\section{Aspectos éticos na pesquisa qualitativa com crianças}

Fecha de recepción: 18 de julio de 2017

Fecha de evaluación: 10 de octubre de 2017

Fecha de aceptación: 31 de octubre de 2017

Disponible en línea: 21 de noviembre de 2017

Luisa Fernanda Moscoso Loaiza*

Luz Patricia Díaz Heredia**

DOI: https://doi.org/10.18359/rlbi.2955

\author{
Cómo citar: \\ Moscoso Loaiza, L. F. y Díaz Heredia, L. P. (2018). \\ Aspectos éticos de la investigación cualitativa con \\ niños. Revista Latinoamericana de Bioética, 18(1), 51-67. \\ Doi: https://doi.org/10.18359/rlbi.2955
}

* Enfermera, especialista en Enfermería Cardiorrespiratoria, Magister en Enfermería, estudiante de Doctorado en Enfermería. Universidad Nacional de Colombia. Becaria de Colciencias Convocatoria 617 Doctorados Nacionales. Bogotá, Colombia. Correo electrónico: lfmoscosol@unal.edu.co. ORCID: http://orcid.org/0000-00020580-1865.

** Enfermera, magister en Enfermería y doctora en Enfermería. Docente de la Universidad Nacional de Colombia, Bogotá. Correo electrónico: lpdiazh@unal.edu.co. ORCID: http://orcid.org/0000-0002-7167-282X. 
Resumen

\section{Abstract}

Resumo
La incorporación de los niños como participantes de estudios investigativos es una práctica más frecuente en la actualidad debido a la necesidad de conocer y entender su visión acerca de las experiencias de vida, lo que plantea nuevos retos y responsabilidades que se centran principalmente en las consideraciones éticas y metodológicas de la investigación. Este artículo describe los aspectos éticos que deben ser tenidos en cuenta en la investigación cualitativa con niños. Es un artículo de revisión con búsqueda sistemática en bases de datos, incluyendo artículos científicos, libros y literatura gris. Asimismo, se abordan las consideraciones éticas que se encuentran implicadas desde la vinculación de los niños como participantes, hasta la obtención de la información y la socialización de los resultados. Para la investigación se seleccionó la información relevante en torno a ocho temas conforme al proceso investigativo. Con esto se evidenció que la cuestión ética constituye un aspecto central en el momento de iniciar y desarrollar cualquier estudio investigativo, por lo que está presente desde su planteamiento hasta su finalización. Pudo concluirse que el cumplimiento de los criterios de rigor metodológico, como la dependencia, credibilidad, transferibilidad y confirmación, asegura la calidad de la investigación cualitativa con niños, y esto permite la adecuada aplicación de los principios éticos de beneficencia y no maleficencia, justicia, autonomía y confidencialidad.

Palabras clave: investigación cualitativa, ética en investigación, metodología, niños.

The incorporation of children as participants in investigative studies is a more frequent practice nowdays due to the need to know and understand their vision about life experiences, which poses new challenges and responsibilities that focus mainly on ethical and methodological considerations of the investigation. This article describes the ethical aspects that should be taken into account in qualitative research with children. It is a review article with systematic search in databases, including scientific articles, books and gray literature. Likewise, we address the ethical considerations involved from the linking of the children as participants to the obtaining of the information and the socialization of the results. For the investigation, the relevant information was selected around eight subjects according to the investigative process. This showed that the ethical issue is a central aspect at the time of initiating and developing any research study, so it is present from its approach to its completion. It's concluded that compliance with criteria of methodological rigor such as dependency, credibility, transferability and confirmation, ensures the quality of qualitative research with children, and this allows the proper application of ethical principles of beneficence and non-maleficence, justice, autonomy and confidentiality.

Keywords: Qualitative research, research ethics, methodology, children.

A incorporação das crianças como participantes em estudos de pesquisa é uma prática mais frequente hoje em dia devido à necessidade de conhecer e compreender sua visão sobre as experiências de vida, o que apresenta novos desafios e responsabilidades que se centram principalmente nas considerações éticas e metodológicas da investigação. Este artigo descreve os aspectos éticos que devem ser levados em consideração na pesquisa qualitativa com crianças. É um artigo de revisão com busca sistemática em bases de dados, incluindo artigos científicos, livros e literatura cinza. Do mesmo modo, são abordadas as considerações éticas que se vem envoltas a partir da ligação das crianças como participantes até à obtenção da informação e à socialização dos resultados. Para a investigação, as informações relevantes foram selecionadas em torno de oito assuntos de acordo com o processo de investigação. Com isto evidenciou-se que a questão ética constitui um aspecto central no momento de iniciar e desenvolver qualquer estudo de pesquisa, pelo que está presente desde a sua abordagem até a sua conclusão. Pode-se concluir que o cumprimento dos critérios de rigor metodológico, como a dependência, credibilidade, transferibilidade e confirmação, garante a qualidade da pesquisa qualitativa com crianças e isso permite a aplicação adequada dos princípios éticos de beneficência e não maleficência, justiça, autonomia e confidencialidade.

Palavras-chave: pesquisa qualitativa, ética em investigação, metodologia, crianças. 


\section{Introducción}

La investigación cualitativa se desarrolla bajo el paradigma naturalista o constructivista, donde la realidad es múltiple y subjetiva, mentalmente construida por los individuos, por lo que existe dentro de un contexto y son posibles muchos constructos. El investigador cualitativo está en constante interacción con el ser investigado; por consiguiente, los hallazgos son creación del proceso interactivo. Las voces e interpretaciones de quienes son estudiados son claves para comprender el fenómeno de interés y las interacciones subjetivas son el principal modo de abordarlos. Los valores y la subjetividad son inevitables y deseables en la investigación cualitativa; por tanto, los procesos inductivos son la mejor forma de obtener evidencia, debido al hincapié que se hace sobre la totalidad del fenómeno de estudio y las interpretaciones basadas en las experiencias de los participantes, ya que buscan la comprensión en profundidad (Polit y Beck, 2014).

Pese a que los supuestos del enfoque cualitativo han girado en torno al participante -quién experimenta y da significado a los múltiples fenómenos de interés-, la investigación en la infancia tendió a evitar la presencia de niños como participantes activos en los estudios. Siendo con frecuencia los adultos, principalmente padres y profesionales, que suministraban las respuestas a las cuestiones que la investigación planteaba en relación con los asuntos específicamente infantiles (Gómez, 2012) (Contreras y Pérez, 2011) (Boyden y Ennew, 2001), condicionando también la práctica clínica, en la cual a menudo se extrapolaban a los niños, los datos obtenidos de estudios de investigación realizados en adultos (Gabaldón, 2012).
La incorporación de los niños como participantes de estudios, tanto cualitativos como cuantitativos, es una práctica más frecuente en la actualidad (Gómez, 2012) (Gabaldón, 2012), debido a la necesidad de conocer y entender la visión que tienen acerca de sus experiencias de vida, que plantea además nuevos retos y responsabilidades a los investigadores, que se centran principalmente en los aspectos éticos y metodológicos por ser considerados.

La cuestión ética constituye un aspecto central al momento de iniciar y desarrollar cualquier estudio investigativo, por lo que debe estar presente desde el planteamiento hasta la finalización y posterior socialización de resultados. En el ámbito de la investigación, la posición de los niños está estructuralmente desprovista en términos de poder, respecto de los adultos investigadores, por lo que "evitar los excesos en el ejercicio de dicho poder debe ser un elemento fundamental a tener en cuenta" (Gómez, 2012, p. 48), y esto se logra a partir del pensamiento reflexivo del investigador en la aplicación y el cumplimiento de los principios éticos, como la beneficencia y no maleficencia, la justicia, la autonomía y la confidencialidad (Fry, Johnstone e International Council of Nurses, 2010).

El pensamiento reflexivo implica reflexividad y esta se refiere a la capacidad de las personas para ser conscientes de sus actos y dar cuenta de ellos (Phillips, 1988), lo que sugiere, "un proceso a través del cual el conocimiento tácito podría convertirse en explícito" (Moore, 2012, p. 67), y compartirse posteriormente con otras personas. El investigador reflexivo es capaz de desprenderse de su quehacer y recapacitar constantemente sobre este, y, por lo tanto, tomar las mejo- 
res decisiones a lo largo de todo el proceso de investigación.

Las decisiones éticas y las opciones metodológicas para trabajar en la investigación cualitativa con infantes están inmersas en las perspectivas y suposiciones ontológicas y epistemológicas, acerca de cómo los investigadores entienden el desarrollo moral y físico del niño o cualquier otro fenómeno relacionado con su bienestar (Gómez, 2012) (Pinto Bustamante y Gulfo Díaz, 2013) (Manguire, 2005) (Graham, Poweell, Taylor, Anderson y Fitzgerald, 2013). Esto incluye la forma como se los conceptualiza, las percepciones que se tienen respecto a su competencia comunicativa, la capacidad en toma de decisiones y su potencial humano, y cómo esto puede y debe ser respetado y representado (Manguire, 2005) (Lidz, Appelbaum, Arnold, Candilis etal., 2012).

A partir de lo expuesto, este artículo tiene como objetivo describir los aspectos éticos que deben ser considerados en la investigación cualitativa con niños, tomando en cuenta las herramientas metodológicas que permiten mostrar y comprender la visión que tienen del mundo. Para el desarrollo de este artículo el concepto de niño hace referencia a los niños y niñas con edades entre los seis y doce años (Descriptores en Ciencias de la Salud (DeCS), 2017), vistos como seres únicos y activos.

\section{Metodología}

La búsqueda bibliográfica se realizó en las bases de datos Science Direct, PubMed y Scielo. Se utilizaron términos de búsqueda como bioethics, ethics, pediatrics, children, research subjects, qualitative research, nursing, incluyendo artículos científicos en idioma inglés, español y portugués, en full text, en el periodo comprendido entre 2006 y 2016, tomando en cuenta algunos clásicos de la psicología infan- til y la metodología cualitativa, así como capítulos de libros y literatura gris. Los artículos científicos fueron seleccionados de forma crítica, a partir de los criterios CASPe 2016.

La información relevante se organizó en torno a ocho temas, conforme al proceso investigativo: 1) invitación a participar de la investigación; 2) la entrevista en la investigación cualitativa; 3) herramientas metodológicas adicionales para la recolección de datos; 4) principio de beneficencia y no maleficencia: los riesgos y beneficios de la investigación; 5) justicia y equidad; 6) respeto por la autonomía: asentimiento y consentimiento informado; 7) confidencialidad y privacidad, y 8) hacer a los niños visibles: difusión de los resultados.

\section{Resultados}

La vinculación de los niños a los estudios investigativos ha traído consigo una gran cantidad de cuestionamientos respecto a si se encuentran o no en la capacidad de entender el contexto de sus decisiones y las posibles consecuencias de estas. Dichos interrogantes han sido abordados principalmente desde la perspectiva del desarrollo moral y cognitivo del niño, para poder establecer un rango de edad aproximado en el que se considere que está en capacidad de discernir y, por ende, que pueda optar por participar o no de una investigación.

Son diversas las teorías propuestas que contradicen o complementan el estudio de la evolución cognitiva del comportamiento del niño, según los postulados clásicos, y que resaltan la influencia de múltiples elementos en la constitución del razonamiento moral, como son las emociones, el papel de estas en la supervivencia evolutiva de la especie humana, la función del afecto y el cuidado, así como del contexto cultural, social y 
simbólico donde se desarrollan las decisiones morales (Harris y Frader, 2011) (Kenny, Downie y Harrison, 2008) (Lindemann y Nelson, 1995) (Córdoba y Acevedo, 2010).

Con base en estas suposiciones, algunos autores consideran el umbral de los doce años como la edad a partir de la cual el niño es capaz de "entender la información y sopesar los riesgos" (Córdoba y Acevedo, 2010). Otros, por su parte, han propuesto que la figura del asentimiento puede darse desde los seis años, ya que en esta edad los infantes están en capacidad de comprender los procesos biológicos o de reflexionar sobre su identidad (Dorn, Susman y Fletcher, 1995) (Nuru-Jeter, Sarsour, Jutte, y Thomas Boyce, 2010).

Desde este contexto, independientemente de la edad del niño, el investigador es responsable de asegurar su vinculación voluntaria, así como su comprensión de la finalidad del estudio y las implicaciones que trae su participación en este; lo cual se alcanza a partir de la incorporación y verificación de los criterios éticos y de rigor metodológico en la investigación cualitativa.

El rigor es un concepto transversal en el desarrollo de un estudio, que permite valorar la aplicación escrupulosa y científica de los métodos de investigación y de las técnicas de análisis para la obtención y el procesamiento de los datos (Hoyle, Harris y Judd, 2002) (Noreña, Alcaraz-Moreno, Rojas y Rebolledo-Malpica, 2012), e implica no solo la adherencia a las normas y reglas establecidas, sino también la preservación y la fidelidad del objetivo del trabajo cualitativo.

Los siguientes criterios deben aplicarse tanto al proceso como al producto, para asegurar el rigor metodológico en la investigación cualitativa (Sandelowski, 1993) (De la
Cuesta-Benjumea, 2011) (Hernández Sampieri, Fernández Collado y Baptista Lucio, 2014):

1. Dependencia-consistencia lógica o estabilidad-auditabilidad: implica que los datos deben ser revisados por distintos investigadores y estos deben arribar a interpretaciones congruentes. Se consideran dos clases de dependencia: a) la interna, que hace alusión al grado en el cual distintos investigadores generan "categorías similares" con los mismos datos, y b) la externa, que se relaciona con el grado en el que diferentes investigadores forman "categorías similares" en el mismo ambiente y periodo, pero cada quien recaba sus propios datos. En ambos casos se trata de verificar la sistematización en la recolección y el análisis cualitativo.

Para asegurar este criterio se deben especificar los juicios construidos en el pensamiento del investigador, detallando cómo y por qué se seleccionan los participantes. Del mismo modo, se tiene en cuenta la reflexividad, por la cual se hacen explícitos los valores y las experiencias del investigador en la toma de decisiones (De la Cuesta-Benjumea, 2011).

2. Credibilidad: se refiere a si el investigador ha captado el significado completo y profundo de las experiencias de los participantes, particularmente de aquellas vinculadas con el planteamiento del problema. Es la correspondencia entre la forma como el niño percibe los conceptos vinculados con el planteamiento y la manera como el investigador retrata sus puntos de vista. Se logra con la inmersión en el campo, al asegurar que los participantes tengan acceso a los resultados de la investigación, y al compararlos con la evidencia de estudios similares o teorías. 
3. Trasferencia o aplicabilidad de resultados: los resultados contribuyen a un mayor conocimiento del significado del fenómeno y al establecimiento de pautas para futuras indagaciones sobre este. Para esto es indispensable la descripción con toda amplitud y precisión del ambiente, los participantes, materiales y momento del estudio. La transferencia nunca será total, solo parcial.

4. Confirmación o confirmabilidad: vinculada a la credibilidad, se refiere a la capacidad de demostrar que se han minimizado los sesgos y las tendencias del investigador. Implica rastrear los datos en su fuente y la explicación de la lógica utilizada para interpretarlos. La inserción al campo, la reflexión sobre los prejuicios, creencias y concepciones del investigador, y la consecución de los criterios anteriores aseguran la confirmación.

El cumplimiento del rigor metodológico no solo asegura la calidad de cualquier investigación cualitativa, sino que además contribuye a la adecuada aplicación de los criterios éticos, los cuales se describen a continuación en concordancia con el proceso investigativo, donde los actores principales son los niños:

\section{Invitación a participar de la investigación}

Uno de los aspectos más relevantes al iniciar una investigación cualitativa es el abordaje de los participantes y la forma como van a ser invitados a tomar parte de esta, sobre todo cuando son niños, ya que se requiere haber obtenido un mayor nivel de confianza para tener un acercamiento más efectivo; esto se alcanza a partir de la inmersión al campo. Munhall (2007) considera que esta permite delimitar el contexto en el marco de la experiencia, y así lo articula a su disponibilidad para la investigación en el momento (Wuest, 2007). Dos dimensiones resultan esenciales con relación al contexto: la conveniencia y la accesibilidad, que deben ser evaluadas a partir de la inmersión, fase que puede variar al pasar el tiempo, dependiendo de las respuestas de los niños ante la presencia de los investigadores y de las observaciones que estos realicen.

La inmersión al campo permite que el investigador se dé a conocer en el ambiente donde va a ser realizada la investigación, y se le facilita el hecho de relacionarse y ganar la confianza con los niños y con las personas a su alrededor (padres, familiares, profesores y profesionales). Adicionalmente, al conocer el contexto, el investigador evalúa la forma más apropiada de acercamiento para informar a los infantes y a los adultos sobre la investigación.

Las metodologías didácticas como las presentaciones de títeres o de videos suelen ser usadas para el abordaje de los niños, mientras que el acercamiento a los padres de familia tiende a realizarse a través de la convocatoria de reuniones por medio de circulares informativas (Kay, Cree, Tisdall, y Wallace, 2003) (Noreña y Cibanal, 2011) (Svendler Nielsen, 2012) (Park, Caine, McConnell y Minaker, 2016). En ambos abordajes es indispensable que el investigador explique el propósito, alcance y procedimiento del estudio, dejando ver la perspectiva ética y la experiencia particular en el trabajo participativo con infantes, demostrando que los resultados esperados de la investigación deben ser de su interés y beneficio.

\section{La entrevista en la investigación cualitativa}

Los niños con más de siete años poseen habilidades para la comunicación verbal y consiguen comprender y expresar sus sentimien- 
tos oralmente, en función de la etapa de desarrollo en la cual se encuentran, lo que facilita el diálogo (Sparapani, Borges, Dantas, Pan y Nascimento, 2012) (Park et al., 2016). Por esto, la utilización de la entrevista como método de recolección de datos es completamente viable en este grupo poblacional.

La entrevista es un diálogo o interacción que se establecen entre el investigador y los participantes (Valles Martínez, 2014), y como tal constituye una herramienta fundamental para la investigación cualitativa. Durante la formulación de las preguntas de la entrevista se sugiere elaborar un guion que abarque los temas relacionados con los objetivos y conceptos del estudio, para obtener información sobre el fenómeno y captar todos sus aspectos, desde la perspectiva de los participantes (Valles Martínez, 2014). La revisión de los conceptos permite que el investigador se enfoque en el propósito y en el fenómeno central de la investigación, para descomponerlo en sus partes.

El investigador debe planificar una introducción a la entrevista, repasando con el niño los objetivos de la investigación, la importancia de conocer su punto de vista, el carácter voluntario de la participación y las cuestiones relacionadas con los principios de la confidencialidad y el anonimato, incluyendo sus limitaciones bajo las legislaciones de protección del infante. Es indispensable utilizar un lenguaje adecuado, formulando las preguntas con la mayor claridad posible, adoptando una escucha activa en relación con estas, respetando lo que se dice y relacionando los temas que aparezcan con otros de interés para la investigación, sin interrumpir, rechazar o adoptar una posición física no preeminente frente al infante (Gómez, 2012). Cuando se hace uso de grabaciones duran- te la realización de las entrevistas, ya sean de audio o video, es necesario dar a conocer a los niños, sus padres o profesores el empleo de este tipo de métodos, informándoles sobre su desarrollo y el modo como se manejará la información recolectada (Noreña et al., 2012).

En relación con el ambiente donde se llevará a cabo la entrevista, a los niños se les debe animar a tomar parte activa en su elección, cuándo prefieren que se realice y si desean o no estar acompañados (Kay et al., 2003), buscando siempre la comodidad del participante y, en lo posible, la menor cantidad de interrupciones. El investigador debe procurar situar a los infantes en una situación protagónica, ya que esto equilibra más el desbalance de poderes existente entre los adultos y los niños (Gómez, 2012), y permite un interés más activo en el trascurso de la entrevista (Kay et al., 2003).

En muchas ocasiones, las entrevistas verbales no promueven la ventaja comunicativa entre el adulto y el infante (Svendler Nielsen, 2012), por lo que utilizar herramientas metodológicas adicionales, como el dibujo, las viñetas visuales o la fotografía permite ampliar el entendimiento de lo que el niño realmente piensa, y esto aumenta su participación por sentirse más libre o menos intimidado (Wagner, 1999).

\section{Herramientas metodológicas adicionales para la recolección de datos}

Usualmente las entrevistas verbales se constituyen en la principal herramienta para la recolección de datos, inclusive en la investigación con infantes; sin embargo, puede ser muy conveniente insertar técnicas adicionales para el logro de los objetivos del estudio. Estas técnicas suelen ser más diverti- 
das que los métodos tradicionales (Kay et al., 2003) (Sparapani et al., 2012), y se considera que pueden mejorar la capacidad del niño para comunicar sus puntos de vista con el investigador, por lo que tienen el potencial de facilitar una comprensión más auténtica de la vida y de sus experiencias (Svendler Nielsen, 2012) (Park et al., 2016) (Greene y Hogan, 2005). Estos esfuerzos imaginativos se han inspirado en un marco interdisciplinario de estudios sobre la infancia que los conceptualiza como intérpretes sociales competentes, cuyas experiencias pueden ser estructuradas a través de sistemas diferentes a los de los adultos (Greene y Hogan, 2005) (Christensen y James, 2008).

Entre estas técnicas encontramos las visuales, donde el participante hace uso de los diferentes procesos cognitivos implicados en la exploración de las imágenes y en el uso del lenguaje para explicar ideas (Kyle, 2015). El hecho de que el procesamiento del lenguaje utilice algunas áreas del cerebro que son diferentes a las del procesamiento de la información visual le da credibilidad a la idea de que los métodos visuales pueden abrir una "forma diferente de conocer y expresar" (Hill, 2013).

Las técnicas visuales pueden plantearse en dos sentidos. En el primero se propone la realización de dibujos por los propios niños, para obtener un testimonio individual de las cuestiones relevantes para la investigación. Es por esto que sus actividades, al igual que sus pensamientos y comportamientos, se pueden identificar cualitativamente mediante el análisis profundo de un dibujo, comprendiendo que los trazos son la forma de empoderamiento del niño sobre el tema por investigar (Wagner, 1999) (Greene y Hogan, 2005).

En el segundo sentido, se trata de conocer las ideas de los entrevistados respec- to a determinados temas a partir de estímulos visuales previamente diseñados por los investigadores, como dibujos, pinturas, fotografías o videos (Gómez, 2012). Las viñetas visuales constituyen un ejemplo de este tipo de estrategias. Una viñeta visual es una historia narrada por un entrevistador, en la que se ponen ejemplos concretos de personas y comportamientos a partir de los cuales los participantes pueden ofrecer comentarios y opiniones, lo que permite al investigador obtener información sobre aspectos importantes en relación a creencias, actitudes y percepciones (Sidiropoulou-Dimakakou, Argyropoulou, Drosos y Terzaki, 2012). Se trata de un pequeño relato construido cuidadosamente con base en experiencias de la vida real (Schoenberg y Ravdal, 2000), en torno a la cual se produce una discusión (Gómez, 2012). Uno de los principales beneficios de esta técnica es que los niños pueden producir sus juicios de una forma despersonalizada (Charles y Manthorpe, 2007), y esto evita la tensión que pudiese existir al hablar de uno mismo (Gómez, 2012).

Otra técnica novedosa es la entrevista fotográfica participativa, en la que se involucra a los niños como colaboradores activos de la investigación, dándoles cámaras e invitándoles a tomar fotos que traten diversos aspectos de su vida (Kyle, 2015) (Jorgenson \& Sullivan, 2010). Los retratos se utilizan posteriormente en el proceso de entrevistas para explorar conjuntamente el significado subjetivo de las imágenes (Jorgenson \& Sullivan, 2010), evocando los comentarios, la memoria, la reflexión y la discusión en el curso de una entrevista semiestructurada. Los ejemplos específicos de interacciones y actividades sociales representadas en las fotos pueden servir de base para una discusión de las 
abstracciones más generales o de los detalles, afinándolos a partir de las mismas (Kyle, 2015).

Sin embargo, el uso de la fotografía presenta desafíos únicos de interpretación porque las imágenes se derivan en gran parte de su importancia desde las circunstancias en las que se producen (Kolb, 2008). A pesar de que estas parecen presentar verdades empíricas sencillas, la creación de retratos por los niños está determinada por factores tales como "su nivel de cualificación, las convenciones de representación pictórica, y también por la interpretación que le dan a la tarea de investigación" (Jorgenson y Sullivan, 2010); por esta razón, el investigador debe esforzarse para participante comprenda los objetivos del estudio y las indicaciones del porqué tomar la fotografía.

\section{Principio de beneficencia y no maleficencia: los riesgos y beneficios de la investigación}

La consideración más importante a la hora de realizar investigaciones con niños es decidir si realmente son necesarias llevarlas a cabo, si deben participar en ellas y cuál será su función. La vulnerabilidad de los menores obliga a ser especialmente exigente en la cobertura de los daños y perjuicios que puedan derivarse de la participación en los estudios (Gabaldón, 2012). En consecuencia, desde el inicio del proceso investigativo se tienen que abordar los elementos críticos en relación con su finalidad, y las repercusiones que su participación pueda tener sobre los niños en lo que se refiere a los daños y beneficios potenciales (Graham etal., 2013).

La beneficencia y la no maleficencia son dos principios éticos involucrados en la acción de procurar el bienestar de los sujetos de la investigación, entendiéndose ambos como actos de bondad que van más allá de la estricta obligación (Mondragón, Monroy, Ito y Medina-Mora, 2010). Actuar sobre los principios de beneficencia y no maleficencia significa ayudar a otros para obtener lo benéfico, involucrarse en conductas para prever o reducir riesgos a los participantes, o ambas (Fry et al., 2010). Incluye el hecho de no hacer daño a otros, acrecentar al máximo los beneficios y disminuir los perjuicios posibles; ambos se traducen como una obligación a todos los involucrados y, por tanto, son anteriores a cualquier tipo de información o consentimiento (Gabaldón, 2012).

En el caso de la investigación científica, los miembros de la sociedad están obligados a reconocer los beneficios y riesgos a largo plazo que puedan resultar del desarrollo del conocimiento y de nuevos procedimientos médicos, psicoterapéuticos y sociales (Mondragón et al., 2010). Entre las formas de riesgos y daños potenciales a los niños se incluyen: el daño físico como consecuencia directa del estudio de investigación propiamente dicho; la represalia, castigo o daño físico infringidos por otras personas debido a su participación en las actividades de la investigación, y el sufrimiento, la ansiedad y la pérdida de autoestima en los estudios de investigación social (Alderson, Morrow y Alderson, 2011) (Carrasco-Aldunate, Rubio-Acuña y Fuentes-Olavarría, 2012).

Existe la posibilidad de que el daño a los niños, sus familias y las comunidades se produzca en el momento de la difusión o comunicación de los resultados. Los principios éticos de beneficencia y no maleficencia son pertinentes aún después de la finalización de la recolección de datos. El daño puede reducirse al mínimo en la etapa de difusión, y mantener la privacidad con respecto a la 
identidad de los participantes (Graham et al., 2013) (Alderson et al., 2011).

\section{Justicia y equidad}

La justicia, entendida como la distribución adecuada del beneficio y obligación (Fry et al., 2010), se refiere al derecho que toda persona posee de ser favorecida con los resultados de la investigación en la que participa, que dicho estudio se realice realmente en el grupo que se requiere investigar y que solo se utilice una población vulnerable cuando en esta sean provechosas las consecuencias (Acevedo Pérez, 2002).

La selección de los niños debe considerar la inclusión de aquellos que pueden beneficiarse de un resultado positivo a corto o largo plazo. Una injusticia ocurre cuando un beneficio al que el niño tiene derecho se niega sin razón válida, cuando se impone una responsabilidad indebidamente o cuando a los iguales se les trata con desigualdad (Fry et al., 2010).

\section{Respeto por la autonomía: asentimiento y consentimiento informado}

La autonomía establece que a los individuos debe permitírseles libertad para determinar sus propias acciones de acuerdo con los planes que hayan elegido; esto implica respetarlos como electores autodeterminados (Fry et al., 2010). La autonomía se cumple cuando existe ausencia de control externo, intencionalidad y conocimiento (Acevedo Pérez, 2002). Una persona autónoma es capaz de deliberar acerca de sus metas personales y de actuar en el sentido de tales deliberaciones, por lo que respetar la autonomía significa dar valor a las opiniones y elecciones, al mismo tiempo que se evita obstruir acciones, a menos que estas vayan claramente en me- noscabo o perjuicio de otros (Carrasco-Aldunate et al., 2012) (Acevedo Pérez, 2002).

La promoción de la autonomía de los niños, a través de su inclusión en los procesos de toma de decisiones, tanto en el ámbito asistencial, como en el campo de la investigación, se logra a través del asentimiento informado. Si bien los niños, dependiendo de su edad y desarrollo cognitivo, no exhiben la capacidad, la madurez y la prudencia para asumir de forma individual el peso absoluto de las decisiones concernientes a su salud y su integridad (Pinto Bustamante y Gulfo Díaz, 2013), sí cuentan con la capacidad de "identificar las reglas morales" (Hersh, Reimer, Paolitto y Hersh, 2002) para asentir participar de una investigación.

Entre los factores que influyen en la toma de decisiones de los niños, se incluyen las experiencias de vida, las vivencias relacionadas con la salud y la enfermedad, la participación en procesos decisionales previos, la influencia externa recibida de otros, la vulnerabilidad condicionada por la dependencia física, emocional y financiera de los adultos, su relativa inexperiencia y la perspectiva limitada ante determinaciones complejas (Wendler, 2006).

El asentimiento informado se constituye, entonces, en un proceso que respeta y promueve el ejercicio a la autonomía en desarrollo del niño, para opinar en los procesos de salud o enfermedad que le afectan; de esta manera, se incentiva en el niño el empoderamiento y el desarrollo de su capacidad moral para el ejercicio autónomo de futuras decisiones (Pinto Bustamante \&y Gulfo Díaz, 2013). El ejercicio de la autonomía requiere elementos previos como la intencionalidad o voluntariedad, la información, la ausencia de coerción y la autenticidad, entendida como la 
coherencia entre los actos de elección y los sistemas de valores particulares (Gracia, Jarabo, Martín y Ríos, 2001).

Según los estándares internacionales de ética (Graham et al., 2013), en la mayoría de países los menores de 16 años de edad, necesitan el consentimiento firmado por parte de sus padres o representantes legales, para la práctica de intervenciones médicas o para la autorización de su inclusión en proyectos de investigación.

El consentimiento informado describe un proceso interactivo en el cual el individuo, o su representante legal en este caso, accede voluntariamente a participar en un estudio, luego de que los propósitos, riesgos y beneficios de este han sido cuidadosamente expuestos y son entendidos por las partes involucradas (Pinto Bustamante y Gulfo Díaz, 2013) (Cabrales-vega et al., 2012) (Marshall, 2007) (Cahana y Hurst, 2008). Por lo que debe proveer información suficiente y de calidad, adecuando la información al nivel de quien la recibirá, evitando tecnicismos y asegurando la voluntariedad de participación (Cabrales-vega et al., 2012) (Noreña et al., 2012) (Marshall, 2007) (Cahana y Hurst, 2008) (Mondragón-Barrios, 2009), insistiendo en que pueden retirarse libremente cuando lo juzguen conveniente, que se guardará confidencialidad de sus datos, que los resultados de la investigación les serán devueltos o retroalimentados, y que su negativa a participar en el estudio no afectará sus derechos (Annas, 2009) (Mfutso-Bengo, Masiye y Adamson, 2008), independientemente del contexto donde se encuentren.

De esta forma, para vincular niños a investigaciones es indispensable contar tanto con su asentimiento, como con el consentimiento de sus padres o responsable legal. El respeto por la autonomía del niño se sustenta también en la responsabilidad del investigador de defen- der el derecho al disentimiento, de negarse a participar y retirarse en cualquier momento de la investigación, y dar prioridad a este derecho por encima de los deseos de sus padres $u$ otras personas (Graham etal., 2013).

\section{Confidencialidad y privacidad}

La confidencialidad se refiere tanto al anonimato en la identidad de los participantes en el estudio, como a la privacidad de la información que es revelada por estos (Noreña et al., 2012). El respeto de la vida privada y la confidencialidad de los niños que participan en la investigación conlleva una detenida reflexión sobre diversos aspectos, como la protección de datos en relación con la cantidad de información que el niño desea revelar o compartir, y con qué persona; y la privacidad en los procesos de recopilación de la información y el almacenamiento de datos que asegure el anonimato de la información intercambiada entre los participantes, a fin de que no puedan ser identificados al publicarse y darse a conocer los resultados (Graham et al., 2013).

El lugar y los métodos utilizados para recoger los datos influyen en la privacidad y confidencialidad; los niños entrevistados deben poder hablar sin ser oídos por personal ajeno a la investigación, y los que proporcionen material escrito o visual deberán hacerlo sin que exista la posibilidad de ser vistos por los demás.

Debe pensarse en el transporte, el almacenamiento y la eliminación de la información, teniendo en cuenta los diferentes formatos de los datos recogidos. Estos formatos pueden ser grabaciones de audio y vídeo, datos escritos a mano y electrónicos (Graham et al., 2013). Los datos personales son confidenciales y al igual que la información obtenida en el estudio, deben ser accesibles únicamente para el investigador o grupo de investigadores, por lo 
que han de guardarse en un lugar cerrado con llave y de acceso controlado, o protegidos por contraseña cuando se trate de archivos informáticos (Shaw, Brady y Ciara Davey, 2011).

La confidencialidad incluye garantizar el anonimato y la imposibilidad de identificar a los participantes de la investigación en los informes, presentaciones y otros medios de difusión de los resultados (Graham et al., 2013) (Shaw et al., 2011), por lo que se puede recurrir a la codificación mediante la sustitución de sus nombres por la asignación de números, o por seudónimos, que pueden ser escogidos por los mismos niños.

Con respecto a la incorporación de otros métodos de recolección de datos, se presentan nuevos retos en relación con la confidencialidad, específicamente con el anonimato. Esto se presenta más que anda en el uso de la fotografía, donde no solo se identifica al niño participante del estudio, sino que también se pueden encontrar vinculadas otras personas, las cuales no han aceptado participar, o peor aún, no tienen conocimiento de la investigación. En estos casos, en lugar de optar por la renuncia a la confidencialidad por parte de los participantes, se opta por usar las imágenes solo como herramientas evocadoras de la memoria, la reflexión y la discusión en el curso de una entrevista a profundidad o semiestructurada (Kyle, 2015), pero no se incluyen como datos para el análisis. De igual forma, el investigador solo puede compartir las fotografías con el equipo de investigación, y en el proceso de análisis, sin poder usarlas en la difusión de los resultados (Kyle, 2015).

Por otra parte, cabe mencionar que existe consenso respecto a que la absoluta confidencialidad no puede ser garantizada, ya que cuando se detecta una amenaza indiscutible que infrinja algún daño en el infante, existe el deber de informar sobre este hecho (Gómez, 2012) (Graham et al., 2013) (Shaw et al., 2011). La garantía de confidencialidad en la información transmitida por los niños está condicionada a que los investigadores no se encuentren con alguna situación anómala (Gómez, 2012); por lo que resulta inapropiado que se consiga su colaboración sin que sean informados de las limitaciones de la confidencialidad (Williamson, Goodenough, Kent y Ashcroft, 2005).

\section{Hacer a los niños visibles: difusión de los resultados}

Los niños son "informantes" competentes y valiosos, que tienen la capacidad de expresar de varias maneras lo que les es importante, teniendo con frecuencia diferentes intereses y puntos de vista de las situaciones en las que los adultos tienen poder sobre ellos (Manguire, 2005). De ahí la importancia de escuchar sus voces y conocer sus opiniones, pensamientos, emociones y perspectivas del mundo social, reconociendo su propia capacidad para la autoreflexividad y toma de decisiones. Para esto se requiere un replanteamiento del concepto de la infancia y una visión más positiva de la agencia y las capacidades de los niños (Manguire, 2005) (Kyle, 2015) (Roth, 2016).

En el proceso de difundir de la información, los investigadores deben mantenerse íntegros y esforzarse por garantizar que los resultados de la investigación sean comunicados con precisión, de forma equitativa, no discriminatoria y que no tergiverse la voz, las experiencias y las circunstancias de los niños (Graham et al., 2013), y asegurar siempre la confidencialidad y privacidad. Asimismo, los menores participantes y sus padres tienen que ser los primeros en conocer los resultados de la investigación. Esta socializa- 
ción de resultados debe procurar hacerse de forma lúdica o dinámica, con adecuación del lenguaje, de tal forma que puedan comprenderla a cabalidad, viendo reflejada su visión.

\section{Conclusiones}

El investigador es responsable de asegurar la vinculación voluntaria de los niños, así como su comprensión de la finalidad del estudio y las implicaciones que trae su participación en este. Esto se alcanza a partir de la incorporación y verificación de los criterios éticos y de rigor metodológico en la investigación cualitativa, y se busca así el mayor beneficio de los niños participantes, por lo que los principios de beneficencia y no maleficencia deben estar por encima de cualquier interés científico o investigativo.

Una de las consideraciones éticas más importantes a la hora de realizar investigaciones con infantes es decidir si realmente es necesario llevar a cabo la investigación, si deben participar en ella y cuál será su función, debido a que la vulnerabilidad de los menores obliga a ser especialmente exigentes en la cobertura de los daños y perjuicios que puedan derivarse de su participación en los estudios.

De igual forma, la adición de nuevas herramientas metodológicas como técnicas para la recolección de datos implica para el investigador la necesidad de ser más cuidadoso con la protección de la confidencialidad y privacidad del niño en relación con la obtención, el almacenamiento de la información y la socialización de los resultados. No se debe olvidar que la finalidad de la investigación cualitativa con niños es hacer visible las perspectivas que tienen del mundo, de sus experiencias y los significados que le otorgan a estas, para poder comprender los procesos sociales de los que son actores fundamentales.

\section{Referencias}

Acevedo Pérez, I. (2002). Aspectos éticos en la investigación científica. Ciencia y Enfermería, 8(1). doi: https://doi.org/10.4067/ S0717-95532002000100003

Alderson, P., Morrow, V. y Alderson, P. (2011). The ethics of research with children and young people: a practical handbook (2da. Ed). Londres: SAGE.

Annas, G. J. (2009). Globalized Clinical Trials and Informed Consent. New England Journal of Medicine, 360(20), 20502053. dpi: https://doi.org/10.1056/NEJMp0901474

Boyden, J. y Ennew, J. (2001). La infancia en el centro de atención: un manual para la investigación participativa con niños. Madrid: Ministerio de Trabajo y Asuntos Sociales, Subdirección General de Publicaciones y Patrimonio Cultural.

Cabrales-Vega, R., Calvachi-Jiménez, J., Foronda-Castro, J., Giraldo-Pinto, D. et al. (2012). ¿Quién se informa con el consentimiento informado? Revista de Salud Pública, 14(3), 502-511.

Cahana, A. y Hurst, S. A. (2008). Voluntary Informed Consent in Research and Clinical Care: An Update. Pain Practice, 8(6), 446-451. doi: https://doi.org/10.1111/ j.1533-2500.2008.00241.x

Carrasco-Aldunate, P., Rubio-Acuña, M. y Fuentes-Olavarría, D. (2012). Consentimiento informado: un pilar de la investigación clínica. Aquichán, 12(1), 32-41. recuperado de http://www.redalyc.org/ html/741/74124091004/

Charles, N. y Manthorpe, J. (2007).

FACS or Fiction? the impact of the 
policy Fair Access to Care Services on social care assessments of older visually impaired people. Practice, 19(2), 143-157. doi: https://doi. org/10.1080/09503150701393692

Christensen, P. M. y James, A. (Eds.). (2008). Research with children: perspectives and practices (2da. Ed.). Nueva York: Routledge.

Contreras, C. y Pérez, A. (2011). Participación invisible: niñez y prácticas participativas emergentes. Revista Latinoamericana de Ciencias Sociales, Niñez y Juventud, 2(9), 811-825. Recuperado de http://www. umanizales.edu.co/publicaciones/campos/cinde/index.html

Córdoba, A. I. G. y Acevedo, D. S. (2010). Consentimiento informado en pediatría. Aplicaciones en psiquiatría. Revista Colombiana de Psiquiatría, 39(4), 758-770. doi: https://doi.org/10.1016/S00347450(14)60214-6

De la Cuesta-Benjumea, C. (2011). La reflexividad: un asunto crítico en la investigación cualitativa. Enfermería Clínica, 21(3), 163-167. doi: https://doi.org/10.1016/j.enfcli.2011.02.005

Descriptores en Ciencias de la Salud (DeCS) (2017). Descriptores en Ciencias de la Salud(DeCS). Recuperado de http://decs. bvsalud.org/E/homepagee.htm

Dorn, L. D., Susman, E. J. y Fletcher, J. C. (1995). Informed consent in children and adolescents: Age, maturation and psychological state. Journal of Adolescent Health, 16(3), 185-190. doi: https://doi.org/10.1016/1054139X(94)00063-K
Fry, S. T., Johnstone, M.-J. y International Council of Nurses. (2010). Etica en la práctica de enfermería: una guía para la toma de decisiones éticas. México, D.F.: El Manual Moderno.

Gabaldón, S. (2012). Aspectos éticos de la investigación en niños y adolescentes. Boletín No. 7,1-8). CEA/CEIC. Recuperado de http://studylib.es/doc/7514934/aspectos-\%C3\%A9ticos-de-la-investigaci\%C3\%B3n-en-ni\%C3\%B1os-y-adolescentes

Gómez, J. M. (2012). El grupo focal y el uso de viñetas en la investigación con niños. EMPIRIA. Revista de Metodología de Ciencias Sociales, (24), 45-66. Recuperado de http://www.redalyc.org/articulo. oa?id=297124737003

Gracia, D., Jarabo, Y., Martín, N. y Ríos, J. (2001). Toma de decisiones en el paciente menor de edad. Medicina Clínica, 115(5), 179-190. Recuperado de http://www. elsevier.es/es-revista-medicina-clinica-2-articulo-toma-decisiones-el-paciente-menor-S0025775301720544

Graham, A., Poweell, M., Taylor, N., Anderson, D. y Fitzgerald, R. (2013). Investigación Ética con Niños / Ethical Research Involving Children (p. 214). Florencia: Centro de Investigaciones de UNICEF Innocenti. Recuperado de http://childethics.com/wp-content/uploads/2015/04/ ERIC-compendium-ES_LR.pdf

Greene, S. y Hogan, D. (Eds.). (2005). Researching children's experience: methods and approaches. Londres, Thousand Oaks: SAGE.

Harris, R. y Frader, J. (2011). Ethical issues in the treatment of pediatric patients with disorders of sex development. En D.S. Diekema, M. R. Mercurio y M. B. Adam 
(Eds.), Clinical ethics in pediatrics: a casebased textbook (pp. 160-165). Cambridge: Cambridge University Press. doi: http:// dx.doi.org/10.1017/CBO9780511740336

Hernández Sampieri, R., Fernández Collado, C. y Baptista Lucio, P. (2014). Metodología de la investigación. México, D.F.: McGraw-Hill Education.

Hersh, R. H., Reimer, J., Paolitto, D. P. y Hersh, R. H. (2002). El crecimiento moral: de Piaget a Kohlberg. Madrid: Narcea.

Hill, J. (2013). Using participatory and visual methods to address power and identity in research with young people. Graduate Journal of Social Science, 10(2), 131-151.

Hoyle, R. H., Harris, M. J. y Judd, C. M. (2002). Research methods in social relations (7ma. Ed.). Fort Worth: Wadsworth.

Jorgenson, J. y Sullivan, T. (2010). Accessing Children's Perspectives Through Participatory Photo Interviews. Forum: Qualitative Social Research, 11(1), 1-23. Recuperado de http://www.qualitative-research.net/index.php/fqs/article/ view/447

Kay, H., Cree, V., Tisdall, K. y Wallace, J. (2003). At the Edge: Negotiating Boundaries in Research with Children and Young People. Forum: Qualitative Social Research, 4(2), 1-22. http://www.qualitative-research.net/index.php/fqs/article/ view/706

Kenny, N., Downie, J. y Harrison, C. (2008). Respectful involvement of children in medical decision making. En P. A. Singer y A. M. Viens (Eds.), The Cambridge textbook of bioethics (pp. 160-165). Cambridge, New York: Cambridge University Press.
Kolb, B. (2008). Involving, sharing, analyzing-Potential of the participatory photo interview. Forum: Qualitative Social Research, 9(3). Recuperado de http://www. qualitative-research.net/index.php/fqs/ article/view/1155/2564

Kyle, M. (2015). Dear Critics: Addressing Concerns and Justifying the Benefits of Photography as a Research Method. Forum: Qualitative Social Research, 16(3), 1-17. Recuperado de http://www.qualitative-research.net/index.php/fqs/article/view/2434

Lidz, C. W., Appelbaum, P. S., Arnold, R., Candilis, P. et al. (2012). How Closely Do Institutional Review Boards Follow the Common Rule? Academic Medicine, 87(7), 969-974. doi: https://doi.org/10.1097/ ACM.0b013e3182575e2e

Lindemann, H. y Nelson, J. L. (1995). The patient in the family: an ethics of medicine and families. Nueva York: Routledge.

Manguire, M. (2005). What if You Talked to $\mathrm{Me}$ I Could Be Interesting! Ethical Research Considerations in Engaging with Bilingual / Multilingual Child Participants in Human Inquiry. Forum: Qualitative Social Research, 6(1), 1-19. Recuperado de http://nbn-resolving.de/ urn:nbn:de:0114-fqs050144.

Marshall, P. L. (2007). Ethical challenges in study design and informed consent for health research in resource-poor settings. Geneva: World Health Organization. Recuperado de http://apps.who.int/iris/bitstream/10665/43622/1/9789241563383_ eng.pdf

Mfutso-Bengo, J., Masiye, F. y Adamson, M. (2008). Ethical challenges in conducting 
research in humanitarian crisis situations. Malawi Medical Journal, 20(2), 46-49. Recuperado de https://www.ncbi. nlm.nih.gov/pmc/articles/PMC3345669/

Mondragón, L., Monroy, Z., Ito, M. E. y Medina-Mora, M. E. (2010). Disyuntivas en las concepciones sobre autonomía y beneficencia que afectan la terapéutica del intento suicida. Acta Bioethica, 16(1). doi: https://doi.org/10.4067/S1726569X2010000100011

Mondragón-Barrios, L. (2009). Consentimiento informado: una praxis dialogica para la investigacion. Revista de Investigación Clínica, 61(1), 73-82. Recuperado de http://www.medigraphic.com/pdfs/ revinvcli/nn-2009/nn091i.pdf

Moore, T. (2012). Keeping them in mind: A reflexive study that considers the practice of social research with children in Australia. (Tesis de doctorado). Australian Catholic University, Canberra. Recuperado de http://researchbank.acu.edu.au/theses/427/

Munhall, P. L. (Ed.). (2007). Nursing research: a qualitative perspective (4ta. Ed.). Sudbury, Mass: Jones and Bartlett.

Noreña, A. L., Alcaraz-Moreno, N., Rojas, J. y Rebolledo-Malpica, D. (2012). Aplicabilidad de los criterios de rigor y éticos en la investigación cualitativa. Aquichán, 12(3), 263-274.

Noreña, A. L. y Cibanal, L. (2011). La experiencia de los niños hospitalizados acerca de su interacción con los profesionales de enfermería. Revista Latino-Americana: Enfermagem, 19(6), 1-8. Recuperado de www.scielo.br/pdf/rlae/v19n6/es_21.pdf
Nuru-Jeter, A. M., Sarsour, K., Jutte, D. P. y Thomas Boyce, W. (2010). Socioeconomic Predictors of Health and Development in Middle Childhood: Variations by Socioeconomic Status Measure and Race. Issues in Comprehensive Pediatric Nursing, 33(2), 59-81. doi: https://doi. org/10.3109/01460861003663953

Park, E., Caine, V., McConnell, D. y Minaker, joanne. (2016). Ethical Tensions as Educative Spaces in Narrative Inquiry. Forum: Qualitative Social Research, 17(2), 1-19. Recuperado de http://www.qualitative-research. net/index.php/fqs/article/view/2571

Phillips, B. (1988). Toward a reflexive sociology. The American Sociologist, 19(2), 13--151. Recuperado de http://connection.ebscohost.com/c/articles/5332249/ toward-reflexive-sociology

Pinto Bustamante, B. y Gulfo Díaz, R. (2013). Asentimiento y consentimiento informado en pediatría: aspectos bioéticos y jurídicos en el contexto colombiano. Revista Colombiana de Bioética, 8(1), 144-165. Recuperado de http://www.redalyc.org/ pdf/1892/189228429010.pdf

Polit, D. F. y Beck, C. T. (2014). Essentials of nursing research: appraising evidence for nursing practice (8va. Ed). Philadelphia: Wolters Kluwer Health, Lippincott Williams \& Wilkins.

Roth, W.-M. (2016). Analyzing the Qualitative Data Analyst: A Naturalistic Investigation of Data Interpretation. Forum: Qualitative Social Research, 16(3), 1-19. Recuperado de http://nbn-resolving.de/ urn:nbn:de:0114-fqs1503119 
Sandelowski, M. (1993). Rigor or rigor mortis: the problem of rigor in qualitative research revisited. Advances in Nursing Science, 16(2), 1-8. Recuperado de https://www.ncbi.nlm.nih.gov/ pubmed/8311428

Schoenberg, N. E. y Ravdal, H. (2000). Using vignettes in awareness and attitudinal research. International Journal of Social Research Methodology, 3(1), 63-74. doi: https://doi. org/10.1080/136455700294932

Shaw, C., Brady, L.-M. y Ciara Davey. (2011). Guidelines for research with children and young people. Londres: National Children's Bureau (NCB) Research Centre. Recuperado de https://www.nfer.ac.uk/ pdf/NCBguidelines.pdf

Sidiropoulou-Dimakakou, D., Argyropoulou, K., Drosos, N. y Terzaki, M. (2012). Career beliefs of Greek and Non-Greek Vocational Education students. Creative Education, 03(07), 1241-1250. doi: https://doi. org/10.4236/ce.2012.37183

Sparapani, V. de C., Borges, A. L. V., Dantas, I. R. de O., Pan, R. y Nascimento, L. C. (2012). Children with Type 1 Diabetes Mellitus and their friends: the influence of this interaction in the management of the disease. Revista Latino-Americana de Enfermagem, 20(1), 117125. doi: https://doi.org/10.1590/S010411692012000100016
Svendler Nielsen, C. (2012). Looking for Children's Experiences in Movement: The Role of the Body in "Videographic Participation". Forum: Qualitative Social Research, 13(3), 1-17. Recuperado de http://www.qualitative-research.net/ index.php/fqs/article/view/1571

Valles Martínez, M. S. (2014). Entrevistas cualitativas (Segunda Edición). Madrid: Centro de Investigaciones Sociológicas.

Wagner, J. (1999). Editor's introduction: Visual sociology and seeing kid's worlds. Visual Sociology, 14(1), 3-6.doi: https://doi. org/10.1080/14725869908583799

Wendler, D. S. (2006). Assent in paediatric research: theoretical and practical considerations. Journal of Medical Ethics, 32(4), 229-234. doi: https://doi.org/10.1136/ jme.2004.011114

Williamson, E., Goodenough, T., Kent, J. y Ashcroft, R. (2005). Conducting research with children: the limits of confidentiality and child protection protocols. Children \& Society, 19(5), 397-409. doi: https:// doi.org/10.1002/chi.852

Wuest, J. (2007). Grounded Theory: the method. En P. L. Munhall (Ed.), Nursing research: a qualitative perspective (4ta. Ed, pp. 239-271). Sudbury: Jones and Bartlett. 